\title{
ARTIGOS
}

\section{DISCUSIÓN Y CONSTRUCCIÓN DE LA CATEGORÍA TEÓRICA DE VULNERABILIDAD SOCIAL ${ }^{1,2}$}

\author{
GUSTAVO DE OLIVEIRA FIGUEIREDO• \\ VALENTINA CARRANZA WEIHMÜLLER • \\ SÔNIA CRISTINA VERMELHO • JUAN BACIGALUPO ARAYA
}

\section{RESUMEN}

El artículo analiza la categoría teórica vulnerabilidad social y presenta resultados de una investigación realizada en favelas de Rio de Janeiro, RJ. A partir de estudios recientes sobre jóvenes, vulnerabilidad y condiciones de vida, problematizamos el concepto de vulnerabilidad social evidenciando y analizando las dimensiones e indicadores que guiaron la recolección de datos (pobreza, exclusión social y violencia). Utilizamos el método de la Investigacíon-Acción-Participación -IAPcontando con la colaboración de educadores que actúan en proyectos sociales en favelas. Los resultados permitieron analizar dos cuestiones: 1) Los abordajes participativos y cualitativos se presentaron fructíferos para la recolección de datos y el análisis de la categoría teórica vulnerabilidad social, 2) La constatación de la categoría exclusión social como condicionante principal de vulnerabilidad, abarcando las dimensiones: social, ideológica, jurídico-legal y extrema/letal.

VULNERABILIDAD • JUVENTUD • VIOLENCIA • EXCLUSIÓN SOCIAL

\section{DISCUSSION AND CONSTRUCTION OF THE THEORETICAL CATEGORY OF SOCIAL VULNERABILITY}

\section{ABSTRACT}

This article analyzes the social vulnerability theoretical category and presents the \section{,}

abor

$$
\text { so }
$$

$$
\text { and }
$$

\section{that} investigation-action-participation - IAP - method. The results enabled us to verify that: 1) the qualitative approaches were positive for data collection and analysis

colaboración de los doctores Albert Bonillo, Adolf Perinat y Conrad Izquierdo, profesores de la Facultad de Psiciología de la Universitat Autónoma de Barcelona, Catalunya. of vulnerability; 2) the confirmation that the social exclusion category is the main condition of vulnerability, encompassing the social, ideological and legal and extreme/lethal dimensions.

VULNERABILITY • YOUTH • VIOLENCE • SOCIAL DISADVANTAGE . 
RÉSUMÉ

Cet article analyse la catégorie de vulnérabilité sociale et présente les résu1tats d'une recherche menée dans les favelas de Rio de Janeiro. Le concept de vulnérabilité sociale est problematisé à partir d'études récentes sur les jeunes et les conditions de vie. Une méthodologie participative RAP avec l'apport d'éducateurs sociaux a permis de mettre en évidence et analyser les dimensions et les indicateurs qui ont guidé la collecte des données (pauvreté, exclusion sociale et violence). Les résultats ont permis d'analyser deux points essentiels: 1) les approches qualitatives ont été positives non seulement pour la collecte des données, mais aussi pour l'analyse de vulnérabilité; 2) le constat que la catégorie exclusion sociale est le principal conditionnant de la vulnérabilité, englobant les dimensions sociale, idéologique, juridique et extrême.

VULNÉRABILITÉ • JEUNESSE • VIOLENCE • EXCLUSION SOCIALE

RESUMO

$O$ artigo analisa a categoria de vulnerabilidade social e apresenta os resultados de uma pesquisa realizada nas favelas do Rio de Janeiro. A partir de estudos sobre jovens, vulnerabilidade social e condições de vida, problematizamos o conceito de vulnerabilidade social, evidenciando e analisando as dimensões e indicadores que nortearam a coleta de dados (pobreza, exclusão social e violência). Utilizamos o método da Investigação-ação participante - IAP -, contando com a participação de educadores de projetos sociais. Os resultados permitiram analisar duas questões: 1) As abordagens qualitativas foram positivas para a coleta de dados e a análise de vulnerabilidade; 2) A constatação da categoria exclusão social como principal condicionante de vulnerabilidade, abrangendo as dimensões social, ideológica, jurídico-legal e extrema/letal. 


\section{SOBRE JÓVENES, JUVENTUDES E VULNERABILIDADES}

- l PARADIGMA COSMOPOLITA EN TORNO A LOS DERECHOS hUMANOS IMPULSÓ EL surgimiento de las minorías, como los grupos sociales específicos que reclamaban sulugar dentro de las sociedades pos Segunda Guerra Mundial. En este contexto, "los jóvenes" se tornaron agentes específicos de protagonismo cultural y político, conformándose como uno de los sectores sociales más contemplados por las dinámicas neoliberales posmodernas.

Como indica Vommaro (2015), los cambios posmodernos han otorgado a los jóvenes un mayor protagonismo, siendo la “juvenilización” de la sociedad una característica fundamental de los movimientos políticos, las dinámicas culturales, los hábitos de consumo, los estilos de vida, la fuerza de trabajo, las sexualidades y los procesos migratorios.

A fin de proponer una definición de "Juventud" acordamos con Abramo (2005) y con el concepto adoptado por la Secretaria Nacional de Juventude de Brasil (BRASIL, 2014) que hablan de la "condición juvenil" como un modo en que una sociedad construye y atribuye significado a "ese momento del ciclo de la vida", o sea, al paso de la infancia a la adultez, siendo el "ser joven”, una dimensión histórico-generacional de tipo transitiva, donde diferentes valores sociales se ponen en juego y se transparentan las diferencias sociales de clase, género, etnia, etc.

La preocupación sobre "las juventudes" también se manifiesta en el aumento de investigaciones que abordan los "problemas que las 
afectan": desempleo, pobreza, mortalidad por causas externas, exposición a violencias de diferentes tipos, delincuencia, adopción de "conductas de riesgo", entre otras. De esta forma, las cuestiones atribuidas a los/las jóvenes no sólo se limitan a factores materiales, sino que se presentan atravesadas por elementos simbólicos ligados a aspectos étnico-raciales, de género, religiosos, territoriales, entre otros. Así, “diversidades e desigualdades son dos de los principales rasgos que pueden caracterizar a las juventudes en la actualidad” (VOMMARO, 2015, p. 13).

Decimos entonces que hoy en día los/las jóvenes ocupan un lugar valorado en las agendas públicas, repercutiendo en el desarrollo de diferentes campos (académico, legal, políticas públicas, consumo, tecnologías, movimientos políticos, cultura, salud, educación).

Como indican Zluhan e Raitz (2014), en su reconstrucción histórica de los estudios sobre juventudes en Brasil, la noción de “juventud” está lejos de un consenso. Según las autoras, es en los años 1990 que las investigaciones sobre el tema incorporan nuevos enfoques, pasando a ver a los jóvenes como "protagonistas de sus propias historias", relativizando enfoques tradicionales asociados a sus “problemas” o “desvíos”. La perspectiva normalizadora de “joven” está tradicionalmente asociada a la mirada "adulta", que ha reafirmado una lectura de joven como "moratoria social”, en el sentido de contemplarlos en su potencialidad: como sujetos que “irán a ser”, restándole relevancia a sus propias inquietudes, prioridades, proyectos y experiencias.

$\mathrm{El}$ avance en los interrogantes y dimensiones que permean a las juventudes actualmente nos permite hablar de su especificidad como grupo diverso e multideterminado por vivencias y procesos intrínsecos al sistema y modo de vida de la modernidad tardía (HARVEY; SOBRAL, 1994), realidad que se presenta compleja por la contradicción entre ampliación de derechos/acceso a bienes simbólicos y la continuidad de restricciones materiales e intensificación de procesos de exclusión/ segregación socioeconómica.

En este sentido, como indican autores latinoamericanos reconocidos en el estudio sobre juventudes (CANCLINI; CRUCES; POZO, 2012; RODRÍGUEZ, 2015; VOMMARO, 2015), las tensiones entre ejercicio de derechos y condiciones de vida atraviesan a muchos jóvenes de sectores populares y periferias de distintas ciudades latinoamericanas, produciendo diferentes subjetividades juveniles. Diversas formas de desarrollo juvenil, participación y compromiso público surgen en escena, a pesar de la emergencia de conflictos urbanos de nuevas magnitudes. En suma: la tensión entre desarrollo/participación y desigualdad/exclusión ha permeado la discusión sobre jóvenes en los últimos años, surgiendo los estudios de vulnerabilidad social como posible campo de abordaje.

En su raíz etimológica, Vulnerable provine del latín vulnerabilis, palabra formada por vulnus (herida) y el sufijo -abilis (-able, que indica 
posibilidad). O sea, vulnerable es aquel que "puede ser herido". Así, vulnerabilidad sería entonces aquella cualidad de "poder ser dañado"; vulnerar, la acción de producir daño o perjuicios y vulneración, el efecto de dañar, agredir. ${ }^{3}$ A partir de este primer acercamiento semántico, podemos entender que vulnerabilidad está asociada a una situación cualitativamente específica (fragilizada) frente a la posibilidad de daño y desestabilización, en la cual un sujeto/agente de vulneración, mediante una relación de poder, coloca a otro sujeto/objeto como vulnerable.

Fue el campo jurídico que la idea de vulnerabilidad aparece relacionada a una "situación de fragilidad"; recordemos las convenciones internacionales sobre Derechos Humanos y el compendio legal por ellas inaugurado, el cual reconoce a las "minorías" como "grupos vulnerables”, siendo los/las jóvenes (menores de edad) incorporados en esta clasificación, justamente por verse limitados en algunos de sus derechos civiles y políticos.

El campo de la Bioética también se apropia de este sentido, cuando establece como grupos vulnerables a niños, niñas y jóvenes a fin de implementar una serie de cuidados especiales respecto a la implementación de tratamientos médicos y consideraciones en la investigación que involucra seres humanos.

A partir de una mirada crítica a estos avances legalistas, Butler (2014) señala que reconocer una situación de vulnerabilidad se ha presentado como una herramienta política para la articulación de luchas de diversos grupos minoritarios, como el caso de los movimientos feministas. De esta forma vemos como, con el surgimiento del paradigma de derechos, el concepto de vulnerabilidad ha ganado fuerza a partir del surgimiento y la consolidación de las llamadas demandas posmodernas y sus movimientos extra-jurídicos. Así, la autora alega por la necesidad de superar el antagonismo de algunas corrientes teóricas y políticas (paternalistas) a fin de comprender vulnerabilidad/resistencia como parte de un mismo proceso,

Una vez que los grupos son etiquetados como 'vulnerables' dentro del discurso de los derechos humanos o los regímenes legales, esos grupos acaban siendo reificados, devienen 'vulnerables' por definición, quedan fijados en una posición de indefensión y falta de agentividad. Todo el poder pertenece al estado y las instituciones internacionales que a día de hoy se supone que han de ofrecerles protección y apoyo. Tales movimientos tienden a infravalorar, o borrar de forma activa, algunos modos de agentividad política y resistencia que emergen dentro de las llamadas poblaciones vulnerables. (BUTLER, 2014, p. 14-15) 
Como dice Butler, vulnerabilidad no estaría asociada a una reificación pasiva y victimista de los grupos subalternos, sino que por el contrario, la consciencia sobre cierta "situación de vulnerabilidad" se presenta como condición necesaria para articular la resistencia.

Por otro lado, en base a los marcos legales anteriormente mencionados, estudios relacionados al Desarrollo Humano han realizado un dislocamiento de la noción originaria de vulnerabilidad, apropiándola como una categoría válida para la comprensión de situaciones de vida limitadas y/o adversas. Surge así la noción de vulnerabilidad social, popularizada por los llamados estudios de vulnerabilidad.

En el campo específico del desarrollo humano y juventudes en América Latina, una de las conceptualizaciones más utilizadas en investigaciones recientes es la propuesta por el informe "Juventud, violencia y vulnerabilidad social en América Latina: desafíos para políticas públicas" (ABRAMOVAY et al., 2002) donde se piensa la vulnerabilidad social como una situación de alcance individual, familiar y/o comunitario asociada a la disponibilidad o privación de tres elementos esenciales: a) recursos materiales o simbólicos; b) oportunidades ofrecidas por el mercado, el Estado y la sociedad; c) las estrategias de uso de los recursos. De esta forma, la vulnerabilidad social como categoría analítica tendría su funcionalidad para el abordaje de grupos sociales que, siendo atribuidos de grandes potencialidades (como el caso de los/las jóvenes por su valor como activo para "el desarrollo"), permanecen, contradictoriamente, "recluidos en escenarios de inseguridad, inestabilidad e marginalidad" (ABRAMOVAY et al., 2002, p. 30). Esta nueva apropiación, de cuño económico desarrollista, ha dado lugar a múltiples investigaciones preocupadas con la "cuestión juvenil" principalmente, respecto al análisis de sus "problemas" para la inserción social y procesos de manutención de desigualdades en sociedades subdesarrolladas.

\section{[...] el enfoque de la vulnerabilidad social constituye una herramienta válida para comprender la situación de los jóvenes, especialmente aquellos de camadas populares, y de su relación con la violencia, ya que a pesar de ser considerados actualmente como actores claves del desarrollo, las estadísticas presentan una realidad mucho menos feliz. ${ }^{4}$ (ABRAMOVAY et al., 2002, p. 31, traducción propia)}

Vemos como esta versión desarrollista de la vulnerabilidad en jóvenes es la que ha cobrado principal relevancia en estudios posteriores interesados en abordar la contradicción entre condiciones de vida y posibilidades de desarrollo humano, pues vulnerabilidad social se presenta como categoría síntesis, o sea, como concepto articulador, para el análisis de aquellos elementos considerados limitantes para "el desarrollo", a

\section{4}

En el original: "[...] o enfoque de vulnerabilidade social constitui ferramenta válida para compreender a situação dos jovens, especialmente aqueles de camadas populares, e da sua relação com a violência já que, apesar de atualmente serem considerados os atores chaves do desenvolvimento, as estatísticas apresentam uma realidade muito 
saber: a) la pobreza, privación/inestabilidad económica; b) la exclusión/ marginalidad y; c) la inseguridad/violencia.

Otras investigaciones recientes han avanzado en la complejización de los análisis de vulnerabilidad, proponiendo otras derivaciones y consideraciones analíticas. En los campos de la salud pública y la salud colectiva el enfoque en torno a la vulnerabilidad, como situación compleja de exclusión, fragilidad y privación presentes en ciertas situaciones, ha sido contrapuesta a la perspectiva de "riesgo", por presentar esta última ciertas limitaciones para comprender a los procesos de salud enfermedad ligados a determinantes sociales y otros condicionantes estructurales, además de producir estigmatizaciones sobre ciertos grupos y comportamientos (AYRES et al., 2008). En este campo también ha sido relevante el esfuerzo en especificar situaciones específicas de vulnerabilidad, sea a enfermedades o agravantes de la salud puntuales (vulnerabilidad a...), sea para profundizar la relación entre sujeto vulnerado e agente/proceso vulneralizador (vulnerabilidad por...). De esta forma, se pasa a hablar de "situaciones de riesgo" a fin de hacer foco en el problema (y no en los individuos) para implementar posibles estrategias de prevención de carácter ambiental y social (CAMAROTTI; KORNBLIT, 2015). El riesgo se comprende así, de manera multideterminada, en relación directa con factores sociales estructurales (condiciones de vida y trabajo, acceso a los servicios públicos, etc.), dejando de ser visto como mero resultado de comportamientos individuales aislados.

Más allá de los esfuerzos académicos realizados desde diferentes áreas, la vulnerabilidad como categoría analítica aún se encuentra en debate. Como definirla teóricamente y a partir de allí, pensar su abordaje metodológico, es todavía una tarea en construcción. Una preocupación constante de los estudios de vulnerabilidad ha sido definir indicadores y procedimientos metodológicos para poder medirla. Recientemente Schumann y Moura (2015) publicaron una revisión de literatura referente a índices sintéticos de vulnerabilidad, en la cual incluyen dos específicos para la medición de vulnerabilidad en juventudes: El Índice de Desarrollo Juvenil -IDJ-, elaborado por la UNESCO (2003) y el Índice de Vulnerabilidad Juvenil -IVJ-, elaborado por la Fundação Seade, São Paulo, Brasil (2002).

El trabajo de las autoras nos permite afirmar que existen pocas y desactualizadas herramientas de medición sintética de vulnerabilidad específicas para jóvenes. De hecho, las mismas autoras indican las siguientes limitaciones para el abordaje analítico de esta categoría: a) la dificultad de representar procesos dinámicos por medio de medidas cuantitativas e sintéticas; b) la indisponibilidad de informaciones necesarias (falencias en los registros, calidad y desagregación de los datos, diferencias por regiones y países, deficiencias presupuestarias para asumir los costos de los estudios); c) la centralidad en los datos 
estadísticos en detrimento de la valorización sobre los conocimientos teóricos para abordar el tema; d) la necesidad de acompañar los resultados con cartografías; e) la poca articulación entre resultados de estudios y la gestión activa en los territorios y la salud pública (SCHUMANN; MOURA, 2015). A pesar de estas limitaciones, muchas investigaciones sobre distintos contextos y grupos de jóvenes han desarrollado diversos métodos para dar cuenta de la vulnerabilidad y sus dimensiones.

Un documento importante publicado en 2015 por la Secretaria Nacional de Juventude, junto al Ministério da Justiça y el Fórum Brasileiro sobre Segurança Pública, en el marco del Plan "Juventude Viva”, fue el informe "O índice de vulnerabilidade juvenil à violência e desigualdade racial 2014" (BRASIL, 2015). En este documento se presentan los resultados a nivel nacional, regional y local sobre jóvenes y vulnerabilidad a la violencia a partir de una estrategia de medición novedosa. El IVJ - Índice de Violencia y Desigualdad Racial incorpora específicamente en la dimensión violencia un indicador de desigualdad racial, expresando la "razón entre la tasa de mortalidad violenta de jóvenes negros y la tasa de mortalidad violenta de jóvenes blancos (BRASIL, 2015, p. 11).

Vemos como este informe propone una nueva estrategia de medición para darcuenta de las “condiciones de vida de la población joven en Brasil, y de cómo el fenómeno de la violencia contra este segmento es afectado por la intervención de múltiples factores socioeconómicos y demográficos, con principal destaque para la influencia de la desigualdad racial” (BRASIL, 2015, p. 12). Así, a partir de un análisis complejo de datos estadísticos públicos, propone abordar indicadores de violencia, relacionándolos con factores sociales e ideológicos también presentes en la dinámica social. La investigación que se presenta a continuación también corrobora esta sinergia.

\section{EL ESTUDIO EMPÍRICO: FAVELAS CARIOCAS, JÓVENES Y VULNERABILIDAD}

El estudio que se presenta fue realizado conjuntamente con 8 Instituciones no gubernamentales, siendo el objeto de investigación los 9 proyectos de intervención social por ellas implementados en 12 favelas de la ciudad de Rio de Janeiro. Los informantes fueron educadores sociales $(\mathrm{n}=18)$, que coordinaban y/o llevaban a cabo las actividades de los proyectos de intervención en las instituciones.

En una primera fase exploratoria, se recolectaron datos cualitativos con un planteamiento abierto y de bajo control interno. En una segunda fase, y a partir de los datos exploratorios, se construyeron cuestionarios altamente estructurados para recoger los datos definitivos. 
Los cuestionarios utilizados en la investigación fueron construidos ad hoc para atender a las situaciones específicas detectadas en las consultas directas y en las observaciones registradas en los cuadernos de campo. Fueron listadas las cuestiones que pudieran favorecer un acercamiento realista y ponderado de los proyectos de intervención implementados por las instituciones consultadas.

En un intenso proceso de reflexión, a partir de la profundización de la experiencia vivida y el acercamiento teórico-metodológico al problema objeto de estudio (la vulnerabilidad social), fueron identificadas las preguntas que componen el cuerpo de contenido de los cuestionarios.

Las preguntas fueron discutidas ampliamente durante el proceso de elaboración de los cuestionarios con expertos académicos y con profesionales relacionados a los proyectos. La intención no fue producir un instrumento estadísticamente estandarizado, sino sensible al carácter evaluativo-formativo y abierto del instrumento en el marco de la Investigación-Acción Participativa -IAP.

Las opciones de respuesta fueron planteadas como escalas de apreciación de cinco niveles (de 0 a 5). El grado de presencia real fue establecido bajo la forma de escala psicométrica con valores variables de 0 a 5. Así, para cada ítem del cuestionario, los informantes elegían el valor correspondiente de acuerdo a su percepción subjetiva. La escala psicométrica representa los grados variables de presencia atribuidos por los educadores, siendo los valores: 0 ("ninguno"), 1 ("muy bajo"), 2 ("bajo"), 3 (“moderado"), 4 (“alto") y 5 (“muy alto"). Los valores escalares constituyen las puntuaciones del grado de presencia atribuido.

Los educadores sociales de cada proyecto de intervención se encontraron en una reunión de trabajo específicamente concebida para contestar colectivamente los cuestionarios. Fue a partir de este dispositivo colectivo que 18 educadores respondieron a las cuestiones a partir del diálogo y la negociación de sus puntos de vista.

En el análisis estadístico se utilizó el programa SPSS para producir el análisis factorial y los análisis complementarios para la crítica y evaluación interna de los indicadores y dimensiones del cuestionario con el objetivo de producir su validación y generar un plan de análisis más adecuado a la realidad práctica. Fueron efectuados testes de Reliability, testes con el Alfa de Cronbach para verificar la fiabilidad de los ítems y las Correlaciones de Pearson para verificar la relación entre los ítems.

Fueron elaboradas tablas de fiabilidad por ítem con el objetivo de verificar y seleccionar las medias estadísticamente relevantes, depurando los descriptores de cada uno de los indicadores y también todos los indicadores de cada una de las dimensiones del estudio. 


\section{RESULTADOS}

Los resultados encontrados fueron agrupados en dos grandes categorías empíricas y analíticas: a) Vulnerabilidad social desde la disponibilidad y calidad de servicios en las favelas y b) Las dimensiones de vulnerabilidad social según la información brindada por los educadores sociales a partir de la aplicación del cuestionario construido colectivamente.

En relación al primer grupo de resultados, íntimamente relacionado al ambiente/contexto del estudio, seguimos Figueiredo (2012, 2016), quién coloca la noción de favela como un concepto dinámico y complejo, que además de referirse a un territorio específico (a aquel caracterizado por condiciones de vida vulnerables, producto del crecimiento urbano desigual e del impacto de la fragmentación socioeconómica del sistema capitalista), conlleva implicaciones culturales e identitarias positivas. En este sentido, si bien los proceso macroestructurales producen impactos negativos en las condiciones de vida de las comunidades de favela, es en el proprio entramado social de dichos espacios donde se abre la lucha por derechos, valorizando formas de asociación, expresiones culturas y estrategias de participación política. No obstante, discursos hegemónicos enfatizan en las particularidades "negativas" de estos contextos a partir de una visión sensacionalista y alarmista de los "problemas" que se construyen simbólicamente como "naturales a las favelas": insalubridad, delincuencia, marginalidad, ilegalidad, caos, violencia, reproduciendo una visión distorsionada, a fin de relacionar pobreza y exclusión con crimen y "barbarie”.

Así como Figueiredo (2008, 2012, 2016), optamos por utilizar el término "favela" por reconocer su importante potencial como recurso político a la hora de las propias comunidades ejercer su derecho de luchar por condiciones adecuadas para su desarrollo y bienestar. Hablar desde la favela significa por un lado reafirmar el nombre, la cultura y la identidad de este territorio, a fin de acentuar las diferencias dentro de la ciudad transluciendo las tensiones desiguales; y por otro lado, estimular la lucha por los derechos, favoreciendo la formación de nuevas asociaciones y el impulso del desarrollo dentro de las comunidades.

A fin de abordar el entramado social de las comunidades, parte del estudio estuvo orientado a mapear los diferentes equipamientos e servicios públicos presentes en los diferentes territorios donde se localizaban los proyectos sociales abordados. De esta forma, a partir de informaciones compartidas mediante las entrevistas con los educadores sociales, fue posible comprender a las favelas como territorios complejos e diversos, donde diferentes dispositivos institucionales $\mathrm{u}$ organizaciones brindan a las comunidades aquellos recursos y servicios públicos socialmente valorados (salud, educación, ocio, cultura, asistencia social, seguridad, etc.). Consideramos que atender a la presencia y características de los servicios disponibles en estos territorios, específicamente respecto a su 
relación con la comunidad a partir de las posibilidades de generar estrategias para la ampliación de derechos, y particularmente, el rol del Estado en estas dinámicas, son aspectos ineludibles para comprender los alcances del concepto de vulnerabilidad social. En este sentido, la investigación averiguó importantes informaciones.

Sobre la existencia de organizaciones culturales no-religiosas, se observó que en 5 favelas existía solamente 1 organización cultural mientras que en otras 5 no existía ninguna asociación, ni organización o institución cultural. Solamente en 2, de las 12 favelas investigadas, existían más de 3 organizaciones culturales actuantes. En relación a otras organizaciones no gubernamentales -ONG- actuando en el territorio, los datos revelaron que no existía una uniformidad entre ellas. En 9 de las 12 favelas se constató la presencia de otras ONGs en presencia variable.

Los servicios de atención a la salud estaban disponibles, no obstante, fueron considerados precarios o insuficientes. Solamente en una de las 12 favelas no existía ninguna unidad de salud pública. Sin embargo, los educadores denunciaron que en 8 favelas el servicio prestado a la población era extremadamente precario. Cabe destacar que en solamente 3 de las 12 favelas investigadas el servicio de salud fue considerado de buena calidad.

Por otro lado, cuando se consideran los servicios públicos esenciales de bienestar y calidad de vida de la población, (agua potable, recolección de residuos, sistema de descarte de residuos patógenos) los educadores relataron que en las favelas investigadas sí estaban cubiertos estos servicios, aunque en muchas de ellas fueron considerados de calidad regular.

Respecto a la oferta de servicios públicos y al proceso de urbanización e vivienda, en todas las favelas investigadas las casas estaban construidas con ladrillos y cemento. Casi todas poseían calles urbanizadas y pavimentadas y en todas ellas existía un sistema de alumbrado público. El principal dato a destacar fue la precariedad de estos servicios, ya sea por su insuficiencia numérica o por su baja calidad.

Cuando se analiza la oferta y calidad de los servicios educativos, fue identificado que en muchas favelas no existían guarderías ni tampoco institutos de educación secundaria. Aunque en casi todas existían escuelas primarias, el sistema de educación fue considerado muy precario en prácticamente todas ellas.

Respecto a la presencia de las mafias del tráfico de drogas, los educadores denunciaron su presencia en 10 de las 12 favelas investigadas y afirman que en 9 de ellas la actuación de las mafias fue agresiva. En el mismo sentido, cuando contestaron acerca de las intervenciones policiales, los educadores afirmaron que hay intervención policial frecuente en 9 de las 12 favelas investigadas y también denunciaron la 
violencia policial cuando afirmaron que la mitad de las intervenciones de la policía se realizó de forma agresiva.

También fue relatado que en 8 de las 12 favelas existían disputas de territorio por diferentes facciones de las mafias, revelando que en la mitad de estos conflictos puede haber habido invasión del territorio por grupos rivales. Como consecuencia de esta situación, verificamos que en 7 favelas existían problemas de movilidad para los habitantes, o sea, algún tipo de dificultad de moverse en el territorio generado por la actuación de las mafias o de la policía.

Adentrando en los aspectos específicos de la violencia, los colaboradores revelaron que sí existía violencia generada por la policía en todas las favelas, excepto en una de ellas. Respecto a la violencia generada por el comando local de la mafia o por grupos mafiosos rivales que disputan el territorio, ella se hizo presente en 9 de las 12 favelas investigadas. Cuando consideramos exclusivamente los casos de asesinato en los episodios de violencia, los datos no varían mucho. En 9 favelas existían casos de muerte provocada por la policía, en 10 favelas casos de muerte provocados por grupos mafiosos rivales y en 8 de las 12 favelas casos de asesinato provocados por el comando local de la mafia.

Las informaciones presentadas revelan una característica también indicada por estudios recientes, en los cuales se evidencia una violencia propia a diversos factores que operan a nivel social más amplio, pero que repercuten de manera particular en las comunidades de favela, territorios atravesados por procesos de vulnerabilidad/exclusión que redimensionan las violencias sistémicas.

En el segundo grupo de resultados, elaborados a partir de la aplicación de los cuestionarios, se preguntó a los educadores sociales sobre la exposición de los jóvenes participantes en los programas a factores de vulnerabilidad. De esta forma fueron analizados tres indicadores: la pobreza, la violencia y la exclusión social.

Al enfocar más detalladamente en los descriptores de las condiciones de pobreza, verificamos que el desempleo, la desocupación y la ausencia de sueldo propio fueron los principales problemas señalados por los educadores, siendo la media de 4,62, es decir, evidenciando un alto de grado de presencia. 
TABLA 1

DESCRIPTORES DE POBREZA. MEDIA DE PRESENCIA Y DESVIACIÓN TÍPICA

\begin{tabular}{l|c|c}
\hline \multicolumn{1}{c|}{ DESCRIPTORES DE POBREZA } & MEDIA & DP \\
\hline Desempleo / desocupación & 4,6 & 0,7 \\
\hline Depresión / ansiedad & 3,5 & 0,9 \\
\hline Vivienda precaria & 3,3 & 1,0 \\
\hline Familia desestructurada & 3,1 & 1,0 \\
\hline Necesidades básicas & 2,9 & 1,0 \\
\hline Hambre / subnutrición & 2,8 & 0,7 \\
\hline Salud debilitada & 2,6 & 0,9 \\
\hline
\end{tabular}

Fuente: Adaptado de la tabla "5.2 Perfil del escenario de pobreza" (FIGUEIREDO, 2012, p. 191)

En un análisis más depurado de los descriptores de las formas de violencia, los educadores contestaron que el prejuicio y la discriminación están presentes en grados muy altos, así como es alta la presencia de casos de homicidios y asesinatos a los que los/las jóvenes se expusieron. También es relevante el grado de presencia (que varía de moderado a alto) de jóvenes que son víctimas de la represión policial (abuso de poder, agresión y/o violación a los derechos humanos).

TABLA 2

DESCRIPTORES DE VIOLENCIA. MEDIA DE PRESENCIA Y DESVIACIÓN TÍPICA

\begin{tabular}{l|c|c}
\multicolumn{1}{c|}{ DESCRIPTORES DE VIOLENCIA } & MEDIA & DP \\
\hline Prejuicio / discriminación & 4,5 & 0,6 \\
\hline Homicidios / asesinatos & 4,0 & 0,8 \\
\hline Represión policial & 3,3 & 1,3 \\
\hline Víctimas de las mafias de drogas & 2,5 & 2,1 \\
\hline Violencia familiar & 2,5 & 1,7 \\
\hline Uso drogas lícitas & 2,3 & 2,2 \\
\hline Agresión / crueldad & 2,0 & 2,3 \\
\hline Uso drogas ilícitas & 1,5 & 2,4 \\
\hline Involucramiento con las mafias de drogas & 1,3 & 1,9 \\
\hline
\end{tabular}

Fuente: Adaptado de la tabla "5.3 Perfil del escenario de violencia” (FIGUEIREDO, 2012, p. 192).

Los resultados aquí expuestos nos permiten afirmar que los educadores colocaron a la exclusión social como el indicador de vulnerabilidad más representativo. En un análisis de los factores que caracterizan a esta dimensión de exclusión social verificamos que la restricción al accesoy/o ausencia de espacios culturales y/o equipamientos públicos para el ocio fue el problema con el más alto grado de presencia. 
TABLA 3

DESCRIPTORES DE EXCLUSIÓN SOCIAL. MEDIA DE PRESENCIA Y DESVIACIÓN TÍPICA

\begin{tabular}{l|c|c}
\hline \multicolumn{1}{c|}{ DESCRIPTORES DE EXCLUSIÓN SOCIAL } & MEDIA & DP \\
\hline Ausencia espacios de cultura / ocio & 4,4 & 0,8 \\
\hline Servicios salud precarios & 4,1 & 0,7 \\
\hline Dificultad de acceso a la prensa & 4,1 & 0,7 \\
\hline Problemas de saneamiento básico & 4,0 & 0,8 \\
\hline Limitaciones de transporte / movilidad & 3,9 & 0,7 \\
\hline Ausencia de formación profesional & 3,9 & 0,9 \\
\hline Ausencia de educación formal & 3,6 & 1,6 \\
\hline Dificultad de acceso tecnología & 3,4 & 1,0 \\
\hline Analfabetismo funcional & 3,4 & 1,0 \\
\hline
\end{tabular}

Fuente: Adaptado de la tabla "5.4 Perfil del escenario de exclusión social” (FIGUEIREDO, 2012, p. 193)

Como se observa en la tabla, también con un grado de presencia alto siguieron inmediatamente la precariedad de los servicios de salud; la limitación y/o imposibilidad de acceso a la información de prensa de calidad y la deficiencia de servicios públicos de saneamiento básico (agua potable, recolección domiciliaria de residuos, servicio de cloacas y alcantarillado).

De acuerdo con los datos obtenidos, es posible afirmar que los educadores valoraron que la pobreza y la exclusión social fueron las condiciones de vulnerabilidad que estuvieron presentes en grado moderado con tendencia al alza, mientras que la violencia fue valorada en bajo grado. Observamos los resultados en la siguiente tabla:

TABLA 4

INDICADORES DE VULNERABILIDAD SOCIAL. MEDIA DE PRESENCIA Y DESVIACIÓN TÍPICA

\begin{tabular}{l|c|c}
\hline \multicolumn{1}{c|}{ INDICADORES DE VULNERABILIDAD SOCIAL } & MEDIA & DP \\
\hline Exclusión Social & 3,9 & 0,7 \\
\hline Pobreza & 3,4 & 0,5 \\
\hline Violencia & 2,6 & 1,1 \\
\hline Alfa de Cronbach = ,640 & & \\
\hline
\end{tabular}

Fuente: Adaptado de la tabla "5.1 Dimensión: vulnerabilidad social percibida" (FIGUEIREDO, 2012, p. 190).

Así, un énfasis especial recae sobre la exclusión social, que presentó la media más alta, lo cual puede ser interpretado como la presencia de alta marginalidad en los/las jóvenes que participaban de los proyectos. Si realizamos un análisis considerando el conjunto de los descriptores de vulnerabilidad social obtenemos los siguientes resultados: 
TABLA 5

DESCRIPTORES DE VULNERABILIDAD SOCIAL. ORDEN, INDICADOR, MEDIA DE PRESENCIA, DESVIACIÓN TÍPICA Y GRADO DE PRESENCIA

\begin{tabular}{|c|c|c|c|c|c|}
\hline ORDEN & INDICADOR & DESCRIPTORES DE VULNERABILIDAD & MEDIA & DP & GRADO \\
\hline 1 & Pobreza & Desempleo / desocupación & 4,6 & 0,7 & \multirow{7}{*}{ ALTO } \\
\hline 2 & Violencia & Prejuicio / discriminación & 4,5 & 0,6 & \\
\hline 3 & Exclusión Social & Ausencia espacios cultura / ocio & 4,4 & 0,8 & \\
\hline 4 & Exclusión Social & Servicios salud precarios & 4,1 & 0,7 & \\
\hline 5 & Exclusión Social & Dificultad de acceso a la prensa & 4,1 & 0,7 & \\
\hline 6 & Violencia & Homicidios / asesinatos & 4,0 & 0,8 & \\
\hline 7 & Exclusión Social & Problemas de saneamiento básico & 4,0 & 0,8 & \\
\hline 8 & Exclusión Social & Limitaciones de transporte / movilidad & 3,9 & 0,7 & \multirow{9}{*}{ MODERADO } \\
\hline 9 & Exclusión Social & Ausencia formación profesional & 3,9 & 0,9 & \\
\hline 10 & Exclusión Social & Ausencia de educación formal & 3,6 & 1,6 & \\
\hline 11 & Exclusión Social & Depresión / ansiedad & 3,5 & 0,9 & \\
\hline 12 & Exclusión Social & Dificultad de acceso tecnología & 3,4 & 1,0 & \\
\hline 13 & Exclusión Social & Analfabetismo funcional & 3,4 & 1,0 & \\
\hline 14 & Pobreza & Vivienda precaria & 3,3 & 1,0 & \\
\hline 15 & Violencia & Represión policial & 3,3 & 1,3 & \\
\hline 16 & Pobreza & Familia desestructurada & 3,1 & 1,0 & \\
\hline 17 & Pobreza & Necesidades básicas & 2,9 & 1,0 & \multirow{7}{*}{ BAJO } \\
\hline 18 & Pobreza & Hambre / subnutrición & 2,8 & 0,7 & \\
\hline 19 & Pobreza & Salud debilitada & 2,6 & 0,9 & \\
\hline 20 & Violencia & Víctimas de las mafias de drogas & 2,5 & 2,1 & \\
\hline 21 & Violencia & Violencia familiar & 2,5 & 1,7 & \\
\hline 22 & Violencia & Uso drogas lícitas & 2,3 & 2,2 & \\
\hline 23 & Violencia & Agresión / crueldad & 2,0 & 2,3 & \\
\hline 24 & Violencia & Uso drogas ilícitas & 1,5 & 2,4 & \multirow{2}{*}{ MUY BAJO } \\
\hline 25 & Violencia & Relación con mafias de drogas & 1,3 & 1,9 & \\
\hline
\end{tabular}

Fuente: Elaboración de los autores, con base en resultados de tesis doctoral (FIGUEIREDO, 2012).

Conforme esta tabla observamos como los descriptores, en términos generales, presentaron grados de presencia homogéneos en cuanto a su indicador. De esta forma los datos obtenidos se mantienen agrupados y ordenados por indicador: exclusión social encabezando la lista, con alto y moderado grado de presencia, pobreza en la zona media entre moderado y bajo grado de presencia y los descriptores de violencia ocupando los últimos lugares, grados bajo y muy bajo. Más allá de esta lectura, ya presentada por la Tabla 1, consideramos interesante prestar atención a aquellos "descriptores atípicos" que se "dislocan" del valor medio del indicador que lo incluye. Nos referimos a desempleo/desocupación, que se presenta como el descriptor de vulnerabilidad más percibido por los educadores, y a tres descriptores de violencia: prejuicio/discriminación, homicidios y asesinatos, (que aparecen con un alto grado de presencia) 
y represión policial (que se disloca para grado moderado). La siguiente tabla indica los valores de estas desviaciones:

TABLA 6

DESCRIPTORES ATÍPICOS RESPECTO A MEDIA DE INDICADOR. DESVIACIÓN

\begin{tabular}{l|c|c|c}
\hline \multicolumn{1}{c|}{ DESCRIPTOR } & MEDIA & $\begin{array}{c}\text { MEDIA DE PRESENCIA } \\
\text { DE INDICADOR }\end{array}$ & DP \\
\hline Prejuicio / discriminación & 4,5 & 2,6 & 1,9 \\
\hline Homicidios / asesinatos & 4,0 & 2,6 & 1,4 \\
\hline Desempleo / desocupación & 4,6 & 3,4 & 1,2 \\
\hline Represión policial & 3,3 & 2,6 & 0,7 \\
\hline
\end{tabular}

Fuente: Elaboración de los autores, con base en resultados de tesis doctoral (FIGUEIREDO, 2012).

Como se puede observar, el valor los tres primeros descriptores se acerca más a los obtenidos por el indicador exclusión social, lo cual nos permite leerlos como parte de ésta. A prejuicio/discriminación lo podemos pensar como un tipo de exclusión cultural-ideológica, desempleo/ desocupación una exclusión socio-económica y homicidios/asesinato la manifestación límite de la exclusión (negación de la vida como exclusión extrema). Analizando los otros descriptores que obtuvieron un alto grado de presencia, podemos decir que la dimensión ideológica-cultural de la exclusión también se incluye en la ausencia de espacios de cultura/ocio, la dificultad de acceso a la prensa; y la exclusión extrema (negación de la vida) también se conecta a los problemas de saneamiento básico y servicios de salud precarios. De esta manera podemos decir que el estudio permitió observar la marcada relación entre vulnerabilidad y exclusión. Una exclusión sofisticada que trasciende su dimensión social e involucra a otros aspectos de la interacción humana. Proponemos así las siguientes dimensiones de exclusión/vulnerabilidad:

\section{GRÁFICO 1}

DIMENSIONES DE EXCLUSIÓN SOCIAL/VULNERABILIDAD

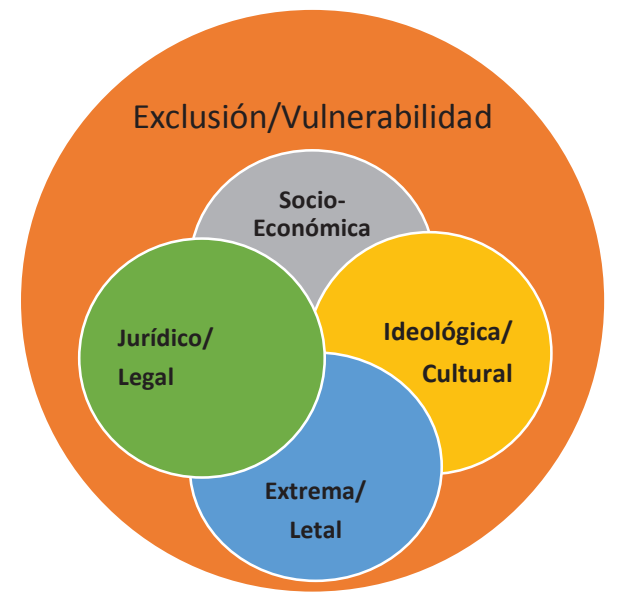


Habiendo sido presentados los resultados, es importante recordar que estos fueron construidos en base a las percepciones de los educadores sociales sobre los jóvenes que viven en las favelas cariocas, información que puede estar condicionada por la relación que ellos mantienen con aquellos jóvenes que frecuentan los programas y proyectos artísticos objetos de investigación. De esta forma, reconocemos la limitación del estudio para incluir informaciones sobre las situaciones de otros jóvenes que, si bien viven y se desarrollan en las favelas de Rio de Janeiro, RJ, no frecuentan este tipo de espacios, y probablemente sus realidades "escaparon" a las reflexiones y respuestas dadas por los educadores.

\section{DISCUSIÓN: REPENSAR LOS FACTORES Y ELEMENTOS DE LA CATEGORIA VULNERABILIDAD SOCIAL}

Enfoques desde el desarrollo humano han realizado principal hincapié en que el combate a la exclusión social se debe realizar a partir de la superación de situaciones de vulnerabilidad a fin de lograr la ansiada “cohesión social”. De esta forma el reconocimiento y combate a la vulnerabilidad social se asumen como una misión pública, que involucra al Estado, sus servicios y políticas, como también a otros agentes civiles. En un artículo reciente Abramovay, Feffermann y Régnier (2012) van a tratar justamente esta cuestión. Reconocen que actualmente las subjetividades juveniles están atravesadas por problemáticas sociales como la violencia, la exclusión, la fragmentación, el individualismo y la valorización del consumo, siendo estos elementos propios al "malestar" contemporáneo. Señalan que ni las políticas públicas, ni las configuraciones del mercado pueden por sí solos superar el problema de la vulnerabilidad social y alegan la necesidad de "fortalecer el capital social intergrupal a través del aumento de la participación y la valorización de las formas de organización y expresión de los propios jóvenes" ${ }^{5}$ (ABRAMOVAY; FEFFERMANN; RÉGNIER, 2012, p. 177, traducción propia).

En base a los resultados del estudio presentado, observamos que la capacidad de los jóvenes para construir su capital social se encuentra fuertemente condicionada por una situación de exclusión que se manifiesta pluricausal en tres planos analíticos: el individual (sujeto), el social (comunidades) y el programático (instituciones) (SILVA et al., 2014). En el estudio presentado observamos que la disponibilidad de los servicios en las favelas es aún deficitaria, además de que muchas

En el original: "[...] pital social intergrupal, através do aumento da participação e valorização das formas de organização e expressão dos jovens". intervenciones por parte del Estado no sólo no generan oportunidades, sino que, por el contrario, las dificultan. La presencia de la policía, limitando la libre circulación de los jóvenes por miedo de sufrir agresiones o abusos de autoridad (inclusive riesgo de muerte) es un elemento claramente identificado por los educadores sociales. 
En este sentido recuperamos el estudio recientemente publicado por Cardoso (2016) sobre datos de homicidios de 2002-2013 disponibles en el sistema de información del Instituto de Segurança Pública -ISP- del Municipio de Rio de Janeiro, RJ. El autor indica que si bien durante el período analizado la tendencia es a una disminución de las tasas de homicidio, desde 2011 a 2013 se ha evidenciado un leve aumento, siendo llamativo en el indicador de personas desaparecidas. Esta constatación levanta la hipótesis que, posiblemente, las formas de violencia en las comunidades de favela de la ciudad de Rio de Janeiro, RJ están adquiriendo nuevas modalidades. Las conclusiones de Cardoso señalan la necesidad de desarrollar estudios cualitativos localizados principalmente en las áreas donde las Unidades de Polícia Pacificadora -UPP- fueron implementadas y de continuar en la articulación y sistematización de acciones entre diferentes sectores gubernamentales y las comunidades (CARDOSO, 2016).

Los resultados presentados también dialogan directamente con la investigación realizada por Camarotti y Kornblit (2015), quienes indican la utilidad del concepto de vulnerabilidades sociales para proponer un modelo integral comunitario para el abordaje de problemáticas sociales. Así afirman que "la vulnerabilidad no es binaria, sino multidimensional y relacional; no es unitaria, siempre hay graduaciones; no es estable, muta constantemente a lo largo del tiempo; las personas no son vulnerables, sino que están vulnerables" (CAMAROTTI; KORNBLIT, 2015, p. 217). Entendiendo a la vulnerabilidad como vulnerabilidades en proceso a partir de diferentes articulaciones entre lo individual y lo social, citan a Delor y Hubert (2000, p. 218) para proponer un abordaje en base a tres dimensiones interrelacionadas: a) trayectorias individuales; b) vínculos e interrelaciones; c) contextos socio-institucionales.

Nuevamente se corrobora el desafío metodológico de cómo obtener aquellas informaciones que nos permitan dar cuenta de los diferentes niveles donde las vulnerabilidades se manifiestan. Consideramos que la opción de incluir a los educadores sociales como colaboradores de investigación, a partir de un trabajo guiado por los principios epistemológicos de la Investigación Acción Participación -IAP-, en articulación con algunos procesos de tratamiento estadístico de datos, es uno de los caminos posibles. En este sentido, consideramos que las informaciones obtenidas pudieron evidenciar, por un lado, las dificultades en medir un objeto que supone múltiples elementos y que se encuentra en permanente dinamismo. La constatación de la centralidad de la dimensión de exclusión evidencia además la necesidad de pensar el concepto de vulnerabilidad desde una perspectiva relacional, dentro de un orden social que se mantiene desigual, donde la pobreza y los procesos de segregación urbana son acompañados de determinantes ideológicos que retroalimentan el status quo dominante. 
Otra cuestión que es importante de colocar en debate es la relación entre vulnerabilidad/exclusión y violencia. En el análisis presentado es posible ver cómo en las dimensiones identificadas dentro de vulnerabilidad/exclusión el componente de violencia se encuentra implícito. La violencia no sólo fue observada en lo que denominamos vulnerabilidad extrema/letal, sino también en el hecho de privar de bienes y servicios (vulnerabilidad socioeconómica), de limitar la agencia política (vulnerabilidad jurídico-legal) y de negar recursos y espacios para el desarrollo cultural y la afirmación simbólica (vulnerabilidad cultural-ideológica). De hecho, como mencionamos al comienzo del artículo, en la misma raíz etimológica del término vulnerabilidad ya se plasma su relación semántica con la de violencia.

Otros estudios también señalan esta relación intrínseca entre estos fenómenos. Rodríguez (2007, p. 546) afirma la necesidad de comprender los crecientes niveles de involucramiento juvenil en algunos de los cuadros de violencia a partir de condiciones de exclusión múltiple en la cual factores económicos, sociales, políticos y culturales se entrelazan.

En Brasil una preocupación creciente es el aumento de la mortalidad por homicidios (causa externa y violenta) en jóvenes hombres negros, quienes están expuestos al homicidio en más del doble $(2,5)$ que los blancos (BRASIL, 2015). El “Mapa de la Violencia 2014” también señala esta problemática pública cuando afirma que "los homicidios son actualmente la principal causa de muerte de jóvenes de 15 a 29 años en Brasil, especialmente en jóvenes negros de sexo masculino, que viven en las periferias y áreas metropolitanas de los centros urbanos" (WAISELFISZ, 2014, p. 9). Los datos disponibles en los registros del Sistema de Salud Brasileño (SIM/Datasus, Ministério da Saúde) evidencian que en 2011 “más de la mitad de las 53.198 muertes por homicidios en Brasil eran jóvenes (27.471, equivalente al 52,63\%), de los cuales 71,44\% fueron negros (pretos y pardos) y 93\% de sexo masculino" (WAISELFISZ, 2014, p. 9).

También Minayo y Constantino (2012) presentan un estudio relevante para abordar el análisis sobre homicidios en municipios de Brasil y Argentina. Las autoras proponen un estudio basado en la teoría de los sistemas complejos, realizando un análisis ecosistémico de la problemática, a fin de relacionar ambiente externo (políticas macrosociales y macroeconómicas), el sistema interno (organización social, gobierno local, participación comunitaria) y el sistema psíquico (la subjetividad) de los habitantes, sea en la construcción de solidaridad o en su responsabilidad por la desintegración social. Vemos como también en este estudio, a pesar de que no se aborde específicamente la cuestión de la vulnerabilidad, la violencia letal (los homicidios) es comprendida a partir del análisis de sus posibles y complejas causas, es decir, de las condiciones (micro-meso-macro) que dan lugar a su ocurrencia. 
Las autoras concluyen en la importancia de la metodología empleada para intentar comprender diversas situaciones en las cuales seguridad, defensa social y respeto a la vida son tensionadas entre dificultades y promoción.

Relacionado al estudio presentado por este artículo, recuperamos la observación de Minayo y Constantino (2012, p. 3277) cuando mencionan que los condicionantes sobre los espacios sociales funcionan como "en un efecto acumulativo": mientras menor es la atención y la disponibilidad de servicios, menor es la seguridad. Así el "el control formal” (dispositivos estatales) llegan siempre posteriormente, como respuesta al aumento de los homicidios y la criminalidad. De esta manera, acordamos con las autoras cuando afirman la sinergia entre ambiente externo, sistema interno y sistema psíquico, siendo todos estos los niveles en los cuales pueden identificarse elementos para comprender la complejidad de los mecanismos y dispositivos que interactúan en los procesos que reproducen la vulnerabilidad/exclusión y su esencial dimensión violenta.

En este mismo sentido, pero con una fuerte pegada de la experiencia practica como educadores sociales, Wimmer y Figueiredo (2006) relatan de forma bastante critica su vivencia en dos favelas de Rio de Janeiro y proponen excelentes elementos para reflexión a partir del concepto de acción colectiva como una potente herramienta para articular las dimensiones teóricas de calidad de vida, autonomía, transdisciplinaridad e Intersetorialidad. De acuerdo con los autores, es en el cotidiano del trabajo que la praxis social transformadora puede establecerse.

\section{CONCLUSIONES}

A fin de concluir este artículo recuperamos tres cuestiones. En primer lugar, consideramos que el estudio presentado se ha propuesto como una estrategia metodológica alternativa para el estudio de las condiciones de vida de los jóvenes y la problematización en torno a la vulnerabilidad. Específicamente señalamos la originalidad en la manera en como los datos fueron siendo construidos: partiendo del diálogo con los educadores para crear los instrumentos de relevamiento de datos, posteriormente generando los espacios para el diálogo y la reflexión conjunta, con las informaciones producidas en estos espacios, pasar para la etapa de tratamiento y sistematización estadística de los datos, y finalmente analizar interpretativamente los resultados obtenidos.

La constatación de la categoría de exclusión como condicionante principal de la vulnerabilidad. Exclusión que no se entiende sólo como concepto socialmente determinado, sino que también supone dimensiones ideológicas, jurídico-legales, e inclusive su forma extrema, 
ligada a un exterminio muchas veces influenciado por ciertas políticas que se consideran de "seguridad" y favorables a una "integración social" de por sí contradictoria.

La necesidad de continuar la línea teórica que concibe a la vulnerabilidad/exclusión como una categoría compleja que supone un abordaje sistémico que permite observar el fenómeno en diferentes niveles de manifestación (maso-meso-micro) entre ellos relacionados.

Invitamos a futuras interrogaciones sobre la posibilidad de pensar a la vulnerabilidad/exclusión desde su dimensión política, retomando los cuestionamientos realizados por Butler (2014), pues como fue posible constatar, la vulnerabilidad, si bien se manifiesta a escala individual, no radica aquí su causa. Las personas no son las vulnerables, sino que se encuentran vulnerabilizadas por contextos que reproducen mecanismos de desigualdad y exclusión social necesarios para el mantenimiento del orden socioeconómico vigente. La cuestión sobre vulnerabilidad/exclusión superaría entonces su interés en simplemente "cómo medirla" desafiando a la pregunta sobre "para qué conocerla".

Finalmente, proponemos algunas cuestiones para reflexión: ¿Es posible pensar la vulnerabilidad/exclusión como identificación motora que, más allá de la integración social, se oriente hacia la generación de cambios y transformaciones estructurales? ¿Es posible pensar la vulnerabilidad/exclusión como herramienta política para el desarrollo de juventudes autónomas y críticamente comprometidas? Invitamos entonces a que en futuras investigaciones que piensen a los jóvenes y sus situaciones de vida se aventuren a indagar sobre este otro horizonte.

\section{REFERENCIAS}

ABRAMO, Helena W. O uso das noções de adolescência e juventude no contexto brasileiro". In: FREITAS, Maria Virginia (Org.). Juventude e adolescência no Brasil: referências conceituais. 2. ed. São Paulo: Ação Educativa, 2005. p. 19-35.

ABRAMOVAY, Miriam; FEFFERMANN, Marisa; RÉGNIER, Jean-Claude. Coesão social e vulnerabilidade no Brasil juventudes e violências. Poiésis: Revista do Programa de Pós-Graduação em Educação, v. 5, p. 165-183, 2012.

ABRAMOVAY, Miriam et al. Juventud, violencia y vulnerabilidad social en América Latina: desafíos para políticas públicas. Brasília, DF: Unesco, 2002. 84 p.

AYRES, Jose Ricardo et al. El concepto de vulnerabilidad y las prácticas de salud: nuevas perspectivas y desafíos. En: CZERESNIA, D.; MACHADO DE FREITAS, C. Promoción de la salud: conceptos, reflexiones y tendências. Buenos Aires: Lugar, 2008.

BRASIL. Secretaria Nacional da Juventude. Índice de vulnerabilidade juvenil à violência e desigualdade racial 2014. Brasília, DF: MJ/SNJ, 2015. 96 p. (Juventude Viva).

BRASIL. Secretaria Nacional de Juventude; ABRAMO, Helena (Org.). Estação juventude: conceitos fundamentais - ponto de partida para uma reflexão sobre políticas públicas de juventude. Brasília, DF: MJ/SNJ, 2014.

BUTLER, Judith. Repensar la vulnerabilidad y la resistencia. In: Simposio de la Asociación Internacional de Filósofas - IAPH, 15., Madrid. Anais... Madrid: IAPH, jun. 2014. p. 24-27. 
CAMAROTTI, Ana Clara; KORNBLIT, Ana Lía. Abordaje integral comunitario de los consumos problemáticos de drogas: construyendo un modelo. Salud Colectiva, v. 11, n. 2, p. 211-221, 2015.

CANCLINI, Néstor García; CRUCES, Francisco; POZO, Maritza Urteaga Castro (Coord.). Jóvenes, culturas urbanas y redes digitales. Madrid: Ariel e Fundación Telefónica, 2012. 302p.

CARDOSO, Francisca Letícia Miranda Gadelha; CECCHETTO, Fátima Regina; CORREA, Juliana Silva; SOUZA, Tiago Oliveira de. Homicídios no Rio de Janeiro, Brasil: uma análise da violência letal. Ciência \& Saúde Coletiva, v. 21, n. 4, p. 1277-1288, 2016. http://dx.doi. org/10.1590/1413-81232015214.14712015.

DELOR, Francois; HUBERT, Michel. Revisiting the concept of "vulnerability". Social Science $\mathcal{E}$ Medicine, v. 50, n. 11, p. 1557-1570, 2000.

FIGUEIREDO Gustavo de O. Desarrollo humano en contextos de exclusión social y violencia: los jóvenes en favelas de Río de Janeiro/Brasil. Tesis [maestría] - Universidad Autónoma de Barcelona, Barcelona, 2008.

FIGUEIREDO, Gustavo de O. Promoción del Desarrollo Humano con Jóvenes en Favelas de Rio de Janeiro/ Brasil: Vulnerabilidad, Resiliencia e Intervención Social. Tese (Doutorado em Psicología) - Universidad Autónoma de Barcelona, Barcelona, 2012. 347 p.

FIGUEIREDO, Gustavo de O. Los jóvenes en favelas de Rio de Janeiro, Brasil: de la vulnerabilidad social a las oportunidades para el desarrollo humano. Revista Ciência \& Saúde Coletiva, v. 21, n. 8. p. 2437-2450, 2016.

HARVEY, David; SOBRAL, Adail Ubirajara. Condição pós-moderna. São Paulo: Loyola, 1994.

MINAYO, Maria Cecília de Souza; CONSTANTINO, Patrícia. Visão ecossistêmica do homicídio. Revista Ciência \& Saúde Coletiva, v. 17, n. 12, p. 3269-3278, 2012.

RODRÍGUEZ, Ernesto. Jóvenes y violencias en América Latina: priorizar la prevención con enfoques integrados. Revista Latinoamericana de Ciencias Sociales, Niñez y Juventud, v. 5, n. 2, p. 539-571, 2007.

RODRÍGUEZ, Ernesto. A modo de prólogo: estudios sobre juventudes en América Latina : Un mosaico de realidades diversas pero convergentes, a caracterizar más y mejor. En: RODRÍGUEZ, et al. Juventudes latinoamericanas: prácticas socioculturales, políticas y políticas públicas. Edición literaria a cargo de Humberto J. Cubides, C. 1. ed. Buenos Aires: CLACSO, 2015. p. 09-20.

SCHUMANN, Lívia Amaral; MOURA, Leides Baroso Azevedo. Índices sintéticos de vulnerabilidade: uma revisão integrativa de literatura. Ciência \& Saúde Coletiva, v. 20, n. 7 , p. 2105-2120, 2015.

SILVA, Maria Angélica Iossi; MELLO, Flávia C. Malta de; MELLO, Débora F. de; FERRIANI, M. das Graças C.; SAMPAIO, Julliane Messias Cordeiro; OLIVEIRA, Wanderlei Abadio de. Vulnerabilidade na saúde do adolescente: questões contemporâneas. Ciência \& Saúde Coletiva, 2014, v. 19, n. 2, p. 619-627, 2014. DOI: 10.1590/1413-81232014192.22312012.

VOMMARO, Pablo. Juventudes y políticas en la Argentina y en América Latina: tendencias, conflictos y desafíos. 1. ed. Buenos Aires: Editor Universitario, 2015.

WAISELFISZ, Julio Jacobo. Os jovens do Brasil: mapa da violência 2014. Brasília, DF: SEPPIR, 2014. (Juventude Viva).

WIMMER, Gert F.; FIGUEIREDO, Gustavo de O.. Ação coletiva para qualidade de vida: autonomia, transdisciplinaridade e intersetorialidade. Revista Ciência e Saúde Coletiva, v. 11, n. 1, p. 145-154, 2006.

ZLUHAN, Mara R.; RAITZ, Tânia R. Juventudes e as múltiplas maneiras de ser jovem na atualidade. Revista de Ciências Humanas, v. 48, n. 2, p. 247-262, 2014.

\section{GUSTAVO DE OLIVEIRA FIGUEIREDO}

Universidade Federal de Rio de Janeiro - UFRJ -, Rio de Janeiro, Rio de Janeiro, Brasil gfigueiredo.ufrj@gmail.com 
VALENTINA CARRANZA WEIHMÜLLER

Universidade Federal de Rio de Janeiro - UFRJ -, Rio de Janeiro, Rio de Janeiro, Brasil cw.valentina@gmail.com

SÔNIA CRISTINA VERMELHO

Universidade Federal de Rio de Janeiro - UFRJ -, Rio de Janeiro, Rio de Janeiro, Brasil cristina.vermelho@gmail.com

JUAN BACIGALUPO ARAYA

Universidade Federal de Rio de Janeiro - UFRJ -, Rio de Janeiro, Rio de Janeiro, Brasil jbacigalupoa@gmail.com 